University of Wollongong

Research Online

Faculty of Engineering and Information

Faculty of Engineering and Information

Sciences - Papers: Part A

Sciences

$1-1-2014$

Rapid assessment of bilateral cochlear implantation for children in Kazakhstan

Lyazzat Kosherbayeva

Asfendiyarov Kazakh National Medical University

David M. Hailey

University of Wollongong, dhailey@uow.edu.au

Laura Kozhageldiyeva

Canadian Society for International Health

Follow this and additional works at: https://ro.uow.edu.au/eispapers

Part of the Engineering Commons, and the Science and Technology Studies Commons

Research Online is the open access institutional repository for the University of Wollongong. For further information contact the UOW Library: research-pubs@uow.edu.au 


\title{
Rapid assessment of bilateral cochlear implantation for children in Kazakhstan
}

\begin{abstract}
Objectives: The aim of this study was to evaluate the effectiveness of bilateral cochlear implantation $(\mathrm{Cl})$ compared with unilateral $\mathrm{Cl}$ for deaf children in the context of the Republic of Kazakhstan health system. Methods. A literature search was conducted, using the PubMed, Cochrane, and Embase data bases for studies that compared the effectiveness of bilateral and unilateral $\mathrm{Cl}$ in children. The search included English language, publications from 2002-2012. Two reviewers independently evaluated all relevant studies. Administrative data relevant to $\mathrm{Cl}$ in Kazakhstan were obtained from the Ministry of Health. Results: Three relevant systematic reviews and an health technology assessment report were found. There was evidence of incremental benefits from bilateral $\mathrm{Cl}$ but the quality of the available studies was poor and there was little information on longer term outcomes. No conclusions could be drawn regarding later incremental improvements to speech perception, learning, and quality of life. To date, in the Republic of Kazakhstan there is not full coverage of audiological screening due to the lack of medical equipment. This leads to late detection of hearing-impaired children and a long rehabilitation period, requiring more resources. Age of implantation in children is late and only a small minority attend general schools. Conclusions: The clinical effectiveness of bilateral $\mathrm{Cl}$, an expensive health technology, requires further study. Given the current situation in Kazakhstan with audiological screening and access to unilateral $\mathrm{Cl}$, there appeared to be other priorities for improving services for children with profound hearing impairment.
\end{abstract}

\section{Keywords}

cochlear, implantation, bilateral, kazakhstan, assessment, rapid, children

\section{Disciplines}

Engineering | Science and Technology Studies

\section{Publication Details}

Kosherbayeva, L., Hailey, D. \& Kozhageldiyeva, L. (2014). Rapid assessment of bilateral cochlear implantation for children in Kazakhstan. International Journal of Technology Assessment in Health Care, 30 (4), 361-365. 


\section{Coversheet}

Title: A rapid assessment of bilateral cochlear implantation for children in Kazakhstan

\section{Short title: Cochlear implantation in Kazakhstan}

\section{Authors}

Lyazzat Kosherbayeva $^{1}$, David Hailey ${ }^{2}$,Laura Kozhageldiyeva ${ }^{3}$

${ }^{1}$ «Republican Centre for Health Development», Astana, Kazakhstan

${ }^{2}$ David Hailey_University of Wollongong

${ }^{3}$ Laura Kozhageldiyeva, Canadian Society for International Health

* Corresponding author:

Lyazzat Kosherbayeva

« Kazakh National Medical University named after S. Asfendiyarov»

90 Tole bi Str,

Almaty, Kazakhstan

Telephone: +77019252228

Lyazzat.k@mail.ru 


\section{Abstract}

Objective: To evaluate the effectiveness of bilateral cochlear implantation (CI) compared to unilateral CI for deaf children in the context of the Republic of Kazakhstan health system.

Methods: A literature search was conducted, using the PubMed, Cochrane and Embase data bases for studies that compared the effectiveness of bilateral and unilateral CI in children. The search included English language, publications from 2002-2012. Two reviewers independently evaluated all relevant studies. Administrative data relevant to CI in Kazakhstan were obtained from the Ministry of Health.

Results: Three relevant systematic reviews and an HTA report were found. There was evidence of incremental benefits from bilateral CI but the quality of the available studies was poor and there was little information on longer term outcomes. No conclusions could be drawn regarding later incremental improvements to speech perception, learning, and quality of life. To date, in the Republic of Kazakhstan there is not full coverage of audiological screening due to the lack of medical equipment. This leads to late detection of hearing-impaired children and a long rehabilitation period, requiring more resources. Age of implantation in children is late and only a small minority attend general schools.

Conclusion The clinical effectiveness of bilateral CI, an expensive health technology, requires further study. Given the current situation in Kazakhstan with audiological screening and access to unilateral CI there appeared to be other priorities for improving services for children with profound hearing impairment.

Keywords: Health technology assessment; Cochlear implantation, Children, Kazakhstan.

\section{Acknowledgements}


The authors of this article express thanks and appreciation for assistance from: Wija Oortwijn (ECORYS Health BV, Rotterdam); Gert-Jan.van der Wilt (Radboud University, Nijmegen); and Vicki Foerster (Canadian Society for International Health). 
Cochlear implantation (CI) is a well - established technology for providing the sensation of sound to people who have profound hearing loss. It requires lengthy rehabilitation and training. Several studies and surveys conducted in different countries show a range of 0.5 to 5 per 1,000 newborns and infants with congenital or early childhood sensorineural hearing loss or severe to profound hearing loss (1). Deaf and hearing-impaired children often have delayed development of speech, language and cognitive skills, which can lead to slow learning and difficulty progressing in school (2). For children who are candidates for CI, implantation at an early age is preferred.

In Kazakhstan, CI for children with profound hearing loss was introduced in 2007 and is carried out by three leading organizations. The State Benefit Package, which provides free medical assistance for citizens of Kazakhstan, covers only unilateral CI. At the request of the patient, bilateral CI may be done but the patient will have to pay additional expenses incurred due to the second implant.

Decision makers within the Ministry of Health of the Republic of Kazakhstan had been presented with the question of whether bilateral CI in children should also be covered under the State Benefit Package. In order to address this issue, information was required on the clinical effectiveness of bilateral CI as compared to unilateral CI.in this patient population.

Responsibility for providing this information was given to the newly - formed Scientific and Practical Centre for Standardization and Health Technology Assessment (CS). The theme was selected by the Department of medical care organization of the Ministry of Health of Republic of Kazakhstan, with CS representatives at the meeting. The team at CS developed a rapid assessment as part of its emerging HTA program, in consultation with ECORYS Health BV (The Netherlands) and the Canadian Society for International Health (3). In this article we present findings from the assessment report and discuss the implications of these for Kazakhstan health care. 


\section{METHODS}

The population considered for the assessment was children under 19 years of age with sensorineural deafness who are candidates for cochlear implantation. The intervention was bilateral CI and the comparator unilateral CI. Outcomes of interest included speech intelligibility and recognition in quiet and in noise, sound localization, oral receptive and expressive language, vocabulary, and communicative competence. Publications eligible for inclusion were systematic reviews, HTAs, and high quality primary studies (preferably RCTs) if these were needed to update the secondary research.

A literature search was conducted, using the PubMed, Cochrane and Embase data bases, for studies that compared the effectiveness of bilateral and unilateral CI in children. The search covered English language publications for the period $2002-2012$. The search strategy is shown in Table 1. Selection of publications and data extraction was undertaken independently by two reviewers and any differences resolved by discussion. The synthesis of study findings drew on the discussion details and summaries of primary studies that were presented in the reviews, and used both quantitative and qualitative data. The quality of the reviews was considered in terms of their information sources, study selection, assessment and synthesis and presentation of results.

Administrative data related to hearing loss in children and the use of CI in Kazakhstan were obtained from the Ministry of Health. Further information on the use of CI in Kazakhstan was obtained in consultation with clinical experts.

\section{RESULTS}

\section{Efficacy of bilateral CI}

The literature search identified 78 titles, 41 of which were not relevant to CI. Of the remaining 37 abstracts, 32 were unrelated to comparison of bilateral and unilateral CI in children, or did not consider clinical effectiveness. Two HTA publications $(4,5)$ and three systematic reviews that 
included comparison of bilateral and unilateral CI in children $(6-8)$ were used for the HTA report. No relevant high quality primary studies were found that had been published subsequent to material included in the reviews..

No randomized controlled trials were identified in the primary evidence base for the reviews. Johnston at al. identified 15 cohort studies, two case-control studies and seven case series. The average sample size of included studies was 12 (range one to 46). Only six studies had 20 or more subjects. (7) The other reviews identified a further four series and a cross-sectional observational study.

The reviews all drew attention to limitations in the quality of available primary studies and variability in the reported primary outcomes (Table 1). Many reports were of cross-sectional studies with participants acting as their own controls. Children who had been bilaterally implanted were tested with either one or both external CI components in place. The quality of the studies varied from moderate to poor (5-8). Forli et al. commented that, with one exception, studies in which the outcomes of children with bilateral CI and unilateral CI were compared had no effective control group (6). Data were mainly gathered retrospectively. Studies were not adequately powered to determine if a difference exists between the children using bilateral CIs compared to unilateral CI. An HTA alert concluded that documentation on the benefits of bilateral CI in children was insufficient. Well-designed, scientific studies were needed to determine whether the method yields positive effects that outweigh the increased risk for complications (4).

The three systematic reviews and the HTA report (5) reached similar conclusions on evidence of effectiveness of bilateral CI (Table 2), finding that this provided incremental benefits when compared to unilateral implantation. Children with bilateral implants had better perception of speech in noisy conditions and greater ability to detect the source of sound. There was also a trend to better perception of speech in quiet conditions, though the differences were not statistically significant. In some but not all studies improvements in speech perception and sound 
localization for children who had bilateral CIs were more consistent for those receiving their second implant within four years of the onset of hearing loss.

There was great diversity in the sorts of tests carried out, test setups, outcome measures and data presentation (8). Forli et al. (6) noted that none of the studies had analyzed the benefits of bilateral vs monolateral CI in terms of language and learning development. Johnston et al. (7) concluded there were additional questions that remain unanswered including: Does the use of bilateral CIs lead to improvements in quality of life compared to use of a single CI in children? What are the perceived parental benefits and risks associated with bilateral cochlear implantation? Which children, in terms of age and severity of loss, benefit from bilateral implantation?, and Will bilateral CIs facilitate the integration of children into the school system?

\section{Use of CI in Kazakhstan}

Between 2007 and 2012 a total of 664 children had received monolateral cochlear implants, with the majority of the procedures (92\%) being carried out at the Republican Children's Clinical Hospital «Aksai» in Almaty. Over this period, 48 per cent of the implants were carried out on children who were over five years old. The proportion of children implanted at less than one year has increased, and had reached 11 per cent of those procedures performed in 2012 (Table 3). Of 395 implanted children for whom schooling details were available, 38 (9,6\% per cent) were in general schools with the rest in schools that cater for those with hearing impairment (Table 4).

During discussion with clinical experts during preparation of the HTA it became apparent that there were many differences between centres in approaches to audiological screening, implantation and rehabilitation. In general there was a lack of common standards, in part due to differences in availability of trained medical personnel and of appropriate equipment.

\section{DISCUSSION}

The effectiveness of CI in children with profound hearing impairment is well established but so far there is no consensus on the status of bilateral CI in children. All of the reviews that we 
identified found there is evidence that bilateral CI in children provides better hearing performance than unilateral CI. Incremental benefits occur through improvement in speech perception and localization of sound sources. However, the extent of improvement has varied between studies and may be modest. A limitation of the rapid HTA was that no formal quality assessment of the systematic reviews was undertaken. However, each of the reviews considered the quality of primary data in some detail. The quality of the available studies is poor and there is little information on longer term outcomes. No conclusions can be drawn regarding later incremental improvements from bilateral CI to speech perception, learning, and quality of life.

This uncertainty regarding the status of bilateral CI has implications for its adoption within Kazakhstan, where the use of CI is still in its early stages. The possible use of a promising extension of the technology has to be considered in the context of current experience in Kazakhstan with CI and related services. Issues informing future decisions include the identification of children who would benefit from implantation, the age of implantation and postimplantation follow up.

Hearing screening programs can give timely identification of children with congenital deafness., giving the opportunity for $\mathrm{CI}$ at an early age. There is good-quality evidence that early detection improves language outcomes (1). Cochlear implantation requires a commitment from the child's carers to long-term involvement in rehabilitation. Complete training may take years, with initial benefit occurring within 6-18 months (5). Early implantation is associated with more rapid rehabilitation and development of communication skills $(5,9)$.

A review initiated by the Ministry of Health in 2012 found that there is limited coverage by audiological screening services in Kazakhstan. Results from 203 medical organizations showed that only $29 \%$ had audiology equipment, so that most centres were unable to undertake screening, leading to loss of early identification of children with hearing impairment. Implantation at an early age has so far been possible for only a small minority of eligible children. Late implantation leads to a long rehabilitation period, and requires more resources. 
Only a small proportion of school - age children who have received implants have attended general schools.

\section{POLICY IMPLICATIONS}

The lack of good information about the incremental clinical benefits from bilateral CI casts doubt on whether this should be covered by the State Benefit Package and provided free of charge to patients. The HTA report from CS concluded that given the current situation with audiological screening and with access to unilateral CI and subsequent rehabilitation there appeared to be other priorities for improving services for children with profound hearing impairment (3). The present focus might well be on increasing coverage of screening services, providing implantation at an earlier age and improving rehabilitation services to increase participation of children with CI in the general school system. Bilateral CI might be an option for the future as further evidence becomes available and the implantation services in Kazakhstan mature.

Despite the fact that CI procedures have been performed in Kazakhstan since 2007, no protocols or clinical guidelines in this area are available. Lack of uniform protocols leads to a fragmented process in which it is easy to lose track of patients who need a CI and of those who have been implanted. Clinical practice guidelines and protocols would provide the basis for a unified approach and resource optimization for all the organizations involved in this area (3).

\section{Decisions by the Ministry of Health}

After considering the CS HTA report on CI, the Ministry of Health decided not to support the provision of bilateral CI implantation for deaf children. Action will be taken to procure equipment for the early detection of children with sensorineural hearing loss, and to strengthen rehabilitation services after CI. A major consideration would have been the need to improve existing CI and associated services as a priority before committing resources to new technology. The uncertain clinical effectiveness of bilateral CI may also have been a factor. 
Also, the Republican Center for Health Development, within the Ministry, has started to coordinate work on the development of clinical guidelines and protocols on CI conjunction with external clinical experts and other health professionals.

\section{REFERENCES}

1.) US Preventive Services Task Force. Universal Screening for Hearing Loss in Newborns: Recommendation Statement. Pediatrics 2008;122; 143-148.

2.) World Health Organization. Newborn and infant hearing screening. Current issues and guiding principles for action. Geneva: WHO Press, 2010. Available at: http://www.who.int/blindness/publications/Newborn_and_Infant_Hearing_Screening_Report.pd $\underline{f}$

3) Kosherbayeva L, Hailey D. Bilateral and unilateral cochlear implantation in children. Astana: Ministry of Public Health of the Republic of Kazakhstan; 2013.

4.) Swedish Council on Technology Assessment in Health Care. Bilateral cochlear implantation (CI) in children (Alert). Stockholm: SBU, 2006.

5.) Bond M, Mealing S, Anderson R, et al. The effectiveness and cost-effectiveness of cochlear implants for severe to profound deafness in children and adults: a systematic review and economic model. Health Technology Assessment 2009;13: No. 44

6.) Forli F, Arslan E, Bellelli S, et al. Systematic review of the literature on the clinical effectiveness of the cochlear implant procedure in paediatric patients. Acta Otorhinolaryngol Ital. 2011; 31(5):281-298.

7.) Johnston JC, Durieux-Smith A, Angus D, Annette O’Connor A, Fitzpatrick E. Bilateral paediatric cochlear implants: A critical review. Int J Audiol 2009; 48: 601 - 617. 
8.) Smulders YE, Rinia AB, Rovers MM, van Zanten GA, Grolman W. What Is the effect of time between sequential cochlear implantations on hearing in adults and children? A systematic review of the literature. Laryngoscope 2011; 121: 1942-1949.

9.) May-Mederake B, Kuehn H, Vogel A, et al. Evaluation of auditory development in infants and toddlers who received cochlear implants under the age of 24 months with the LittlEAR S1 Auditory Questionnaire. Int J PedOtorhinolaryngol 2010; 74:1149-1155. 